\title{
Energy Detection of Multilevel PAM Signals with Systematic Threshold Mismatch
}

\author{
Antti Anttonen, Adrian Kotelba, and Aarne Mämmelä \\ VTT Technical Research Centre of Finland, P.O. Box 1000, 90571 Oulu, Finland \\ Correspondence should be addressed to Antti Anttonen, antti.anttonen@vtt.fi \\ Received 3 December 2008; Accepted 13 February 2009 \\ Recommended by Luca De Nardis
}

\begin{abstract}
We address a symbol decision problem with spectrally efficient energy detected multilevel pulse amplitude modulated (PAM) signals. First, we analytically quantify the relationship between a systematic threshold mismatch and the required increase of the average signal-to-noise ratio to preserve a desired symbol error rate. For the case in which such an increase is not tolerable, we present a novel near-optimal multilevel threshold selection scheme, which is accurate for a wide range of system parameters.
\end{abstract}

Copyright (C) 2009 Antti Anttonen et al. This is an open access article distributed under the Creative Commons Attribution License, which permits unrestricted use, distribution, and reproduction in any medium, provided the original work is properly cited.

\section{Introduction}

The optimal symbol decision rule in an energy detection system with pulse amplitude modulated (PAM) symbols reduces to the problem of finding proper decision threshold values $[1,2]$. There are two kinds of sources for a threshold mismatch. Firstly, a purely random type of threshold mismatch is caused by a possible estimation error of the signal and noise energies required for the threshold selection. Secondly, a more predictable systematic threshold mismatch with respect to the received signal-to-noise ratio (SNR) results from the inability to define the optimal thresholds in a closed form even if the SNR is perfectly known. The former mismatch can be removed with a proper averaging whereas the latter mismatch can be more difficult to compensate.

The effect of a systematic threshold mismatch on the error probability of an energy detected on-off keying (OOK) system has been studied, for example, in [3]. Gaussian approximations for the distribution of an energy detected decision variable with different assumptions of the expected mean and variance are frequently used to reduce the complexity related to the exact error analysis and threshold selection $[4,5]$. These approximations are based on specific assumptions on the value of degrees of freedom (DOFs) reducing their accuracy and robustness, for example, in adaptive multiband systems [6] in which the DOFs can change adaptively or be relatively low. Another approximation for a binary OOK using a numerical approach is suggested in [6]. However, to the best of our knowledge, none of the papers address the effect of the threshold mismatch or accurate threshold selection for a spectrally efficient energy detected PAM (ED-PAM) system to allow arbitrary selection of the number of modulation levels and DOFs.

The novelty of our contribution is two fold. Firstly, after describing our system in Section 2, we analytically quantify the required increase of the SNR per bit to tolerate a hypothesized threshold mismatch for a given error probability in Section 3. Secondly, in Section 4 we propose a new multilevel threshold selection scheme for an ED-PAM system. Since the symbol decision problem of an energy detector in a slowly fading channel without interference reduces instantaneously to that of an additive white Gaussian noise (AWGN) channel [7], for brevity we restrict our channel to be an AWGN channel. We focus on the systematic threshold mismatch of an ED-PAM system and assume perfect estimates for the signal and noise energies.

\section{System Modeling}

The decision variable of a multilevel $M$-ary ED-PAM system can be expressed as

$$
y\left(t_{0}\right)=\int_{t_{0}}^{t_{0}+T_{i}}\left[\int_{-\infty}^{+\infty} r(t-\tau) g(\tau) d \tau\right]^{2} d t
$$


where $r(t)=s(t)+n(t)$ is the received signal, $s(t)=$ $\sum_{k} a_{k} u\left(t-k T_{r}\right)$ is the transmitted signal, $u(t)$ is the pulse waveform, $a_{k} \in\left\{0, \sqrt{E_{1}}, 2 \sqrt{E_{1}}, \ldots,(M-1) \sqrt{E_{1}}\right\}$ is the $k$ th data symbol from the nonnegative and real PAM constellation set, $E_{1}=E_{b} / c_{b}$ is the received energy corresponding to the minimum nonzero amplitude, $E_{b}$ is the average energy per bit, $c_{b}=\left(2 M^{2}-3 M+1\right) /\left(6 \log _{2} M\right), M=2^{b}$ is the number of modulation levels, $b$ is the number of bits per symbol, $T_{r}$ is the symbol period, $n(t)$ is the zero mean AWGN component with noise power spectral density $N_{0}, t_{0}$ is the known start time of the symbol of interest, $T_{i}$ is the integration time, and $g(t)$ is the impulse response of the receiver filter with bandwidth $B$. The product of the integration time and bandwidth is denoted by $D=B T_{i}$, and $2 D$ is the DOFs. It is assumed that $B$ and $T_{i}$ are selected so that $D$ is an integer. The OOK system is a special case of the nonnegative PAM system with $M=2$. The received energy of the $m$ th symbol is $E_{m}=m^{2} E_{1}(m=0,1,2, \ldots, M-1)$. We assume that all symbols are equally probable and that the noise samples affecting the decision variable are uncorrelated. The average SNR per bit is defined as $\gamma=E_{b} / N_{0}$. We use the maximum likelihood decision criterion and multiple hypothesis testing approach [8, page 82]. We make a hypothesis $H_{m}(m=$ $0,1,2, \ldots, M-1)$ that the symbol $a_{m}$ was transmitted if

$$
p\left(y \mid H_{m}\right)>p\left(y \mid H_{k}\right) \quad \forall k \neq m,
$$

where in our case $p\left(y \mid H_{m}\right)$ is the conditional central $(m=0)$ or noncentral $(m>0)$ chi-squared probability density function (pdf) given in [2]. According to the maximum likelihood criterion (2), the optimal symbol decision threshold values $\rho_{m, \mathrm{opt}}<\rho_{m+1, \mathrm{opt}}(m=0,1,2, \ldots, M-2)$ can be found from $p\left(y \mid H_{m}\right)=\left.p\left(y \mid H_{m+1}\right)\right|_{y=\rho_{m, \text { opt }}}$, which is not possible to solve in a closed form.

\section{Performance with Systematic Threshold Mismatch}

The symbol error rate (SER) of the target system can be expressed as [2]

$$
\begin{aligned}
P_{M}\left(D, \gamma, \rho_{0}^{\prime}, \rho_{1}^{\prime}, \ldots, \rho_{M-2}^{\prime}\right) & \\
=\frac{1}{M}[ & M-1+Q_{D}\left(0, \sqrt{2 \rho_{0}^{\prime}}\right) \\
& -Q_{D}\left(\sqrt{2(M-1)^{2} c_{b}^{-1} \gamma}, \sqrt{2 \rho_{M-2}^{\prime}}\right) \\
& -\sum_{m=1}^{M-2} Q_{D}\left(\sqrt{2 m^{2} c_{b}^{-1} \gamma}, \sqrt{2 \rho_{m-1}^{\prime}}\right) \\
& \left.+\sum_{m=1}^{M-2} Q_{D}\left(\sqrt{2 m^{2} c_{b}^{-1} \gamma}, \sqrt{2 \rho_{m}^{\prime}}\right)\right],
\end{aligned}
$$

where $\rho_{m}^{\prime}$ is the selected $m$ th threshold $(m=0,1,2, \ldots, M-$ 2) normalized by $N_{0}$ and $Q_{v}(\cdot, \cdot)$ is the generalized $v$ th order Marcum $Q$-function defined in [9]. To theoretically evaluate the relation between a threshold mismatch and

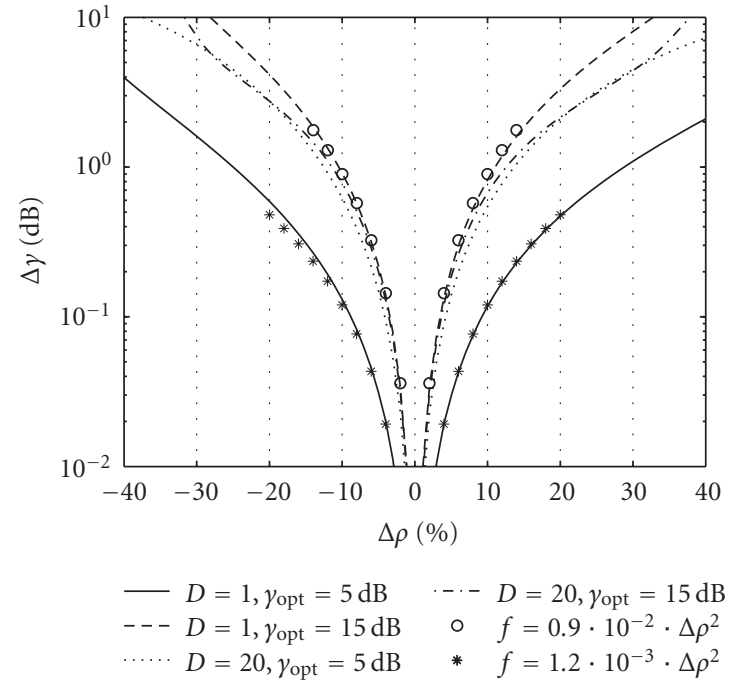

FIGURE 1: Required SNR margin versus threshold mismatch $(M=$ 4).

the corresponding margin in the required SNR per bit to preserve a desired SER, we set

$$
\begin{aligned}
& P_{M}\left(D, \gamma_{\mathrm{opt}}, \rho_{0, \mathrm{opt}}^{\prime}, \rho_{1, \mathrm{opt}}^{\prime}, \ldots, \rho_{M-2, \mathrm{opt}}^{\prime}\right) \\
& \quad=P_{M}\left(D, \gamma_{\mathrm{mis}}, \rho_{0, \mathrm{mis}}^{\prime}, \rho_{1, \mathrm{mis}}^{\prime}, \ldots, \rho_{M-2, \mathrm{mis}}^{\prime}\right),
\end{aligned}
$$

where now $\gamma_{\text {opt }}$ is the SNR per bit to achieve the desired $P_{M}$ with the normalized optimal thresholds $\rho_{m, \mathrm{opt}}^{\prime}$ and $\gamma_{\mathrm{mis}}=$ $\gamma_{\text {opt }} \Delta \gamma$ is the required SNR per bit to achieve the desired $P_{M}$ with the mismatched thresholds $\rho_{m, \text { mis }}^{\prime}=\rho_{m, \mathrm{opt}}^{\prime},\left(1+\Delta \rho_{m}\right)$. In other words, $\Delta y$ denotes the required SNR margin per bit resulted from the hypothesized relative threshold offset $\Delta \rho_{m}$ compared to the corresponding optimal thresholds. We define the above relationship formally as

$$
\Delta \gamma=f\left(\Delta \rho_{m}\right), \Delta \gamma \geq 1,\left|\Delta \rho_{m}\right|<1, m=0,1,2, \ldots, M-2,
$$

where $f(\cdot)$ denotes a functional relation between $\Delta \gamma$ and $\Delta \rho_{m}$ and it depends on selected values of $M, D$, and $\gamma_{\text {opt }}$. It is convenient to express $\Delta \gamma$ in decibels by $\Delta \gamma=$ $10 \log _{10}\left(\gamma_{\text {mis }} / \gamma_{\text {opt }}\right) \mathrm{dB}$ and $\Delta \rho_{m}$ in percentages by $\Delta \rho_{m}=$ $100 \cdot\left(\rho_{m, \mathrm{mis}}^{\prime} / \rho_{m, \mathrm{opt}}^{\prime}-1\right) \%$. There is no closed form solution for (5) from (4). We use the numerical bisection method described in [10, page 261] to find the relationship and some examples are given in Figure 1. Assuming the offset $\Delta \rho=$ $\Delta \rho_{m}$ in percentage is the same for all $m$, we suggest that a simple quadratic function with a single parameter can be used to approximate (5) with a good accuracy for relatively low values of $\Delta \rho$ and $\Delta \gamma$. The parameter of the quadratic function depends on the selected values of $M, D$, and $\gamma_{\mathrm{opt}}$ and it can be found numerically. 


\section{Proposed Near-Optimal Threshold Selection Scheme}

To solve the decision threshold selection problem we propose to find an error function between the optimal and asymptotically approximated threshold values, that is, when the SNR approaches infinity. A similar approach was first proposed in [6] without detailed evaluation for an energy detection system with binary OOK. However, the problem of finding an error function for a multilevel ED-PAM system is evidently more complicated. First, the asymptotically optimal values $\rho_{m \text {,asy }}$ should be found and they are derived differently for the threshold values $\rho_{0 \text {,asy }}$ and $\rho_{m \text {,asy }}$ with $m>0$. It is shown in [6] that $\rho_{0 \text {,asy }}=E_{1} / 4$ when the SNR approaches infinity. However, we observed that the corresponding result for $\rho_{m \text {,asy }}$ with $m>0$ is much more complicated (the result is not shown here due to space limitations). We alternatively assume that for a high SNR, the mean value of the decision variable is unaffected by the noise and the decision variable follows a symmetrical distribution, which directly results in a simple approximation $\rho_{m \text {,sym }}=\left(E_{m}+E_{m+1}\right) / 2, m>0$. The overall scheme is referred to as a semiasymptotically optimal scheme since only $\rho_{0 \text {,asy }}$ is asymptotically unbiased whereas there will be some bias in $\rho_{m \text {,sym }}$ with $m>$ 0 even if the SNR approaches infinity. The second step is introduced to find an error function $e_{m}$ between the optimal and semiasymptotically optimal threshold value set mentioned above. After some manipulations we propose to minimize

$$
e_{m}=\left\{\begin{aligned}
& \min \left\{\frac{1}{N_{D}} \sum_{i=0}^{N_{D}-1} \frac{\rho_{0, \mathrm{opt}} \alpha-E_{1} / 4-d_{2,0}(i)}{d_{1,0}(i)}\right\}, \quad m=0, \\
& \min \left\{\frac{1}{N_{D}}\right. \\
&\left.\times \sum_{i=0}^{N_{D}-1} \frac{\rho_{m, \mathrm{opt}} \beta-\left(E_{m}+E_{m+1}\right) / 2-d_{2, m}(i)}{d_{1, m}(i)}\right\}, \\
& m>0,
\end{aligned}\right.
$$

where $\alpha$ denotes $\left[0, E_{1} / N_{0}, R_{D}(i)\right]$ and $\beta$ denotes $\left[E_{m} / N_{0}\right.$, $\left.E_{m+1} / N_{0}, R_{D}(i)\right]$ and $R_{D}(i)=i+1\left(i=0,1, \ldots, N_{D}-1\right)$ is the $N_{D}$-element value set to be supported for the parameter $D$, $d_{1, m}$, and $d_{2, m}$ are the first-order coefficients, and $\rho_{m, \text { opt }}(\cdot, \cdot, \cdot)$ are the optimal thresholds depending on the particular value of $D$ and the SNRs of the symbols. An analytical closed form solution to the above problem cannot be found. In order to make the error function parameterized by $D$, its relation to the error function is found experimentally with the help of the first-order coefficients $d_{1,0}(i)=N_{0} \sqrt{R_{D}(i)-1}$ if $m=$ $0, d_{1, m}(i)=N_{0}$ if $m>0$, and $d_{2, m}(i)=R_{D}(i) N_{0}$ for $m \geq 0$. The remaining error is averaged over the selected range of the expected values of $D$. We select a polynomial-based error function with the aim to maintain low complexity. Starting from (6), we obtain, after some manipulations, a novel data-

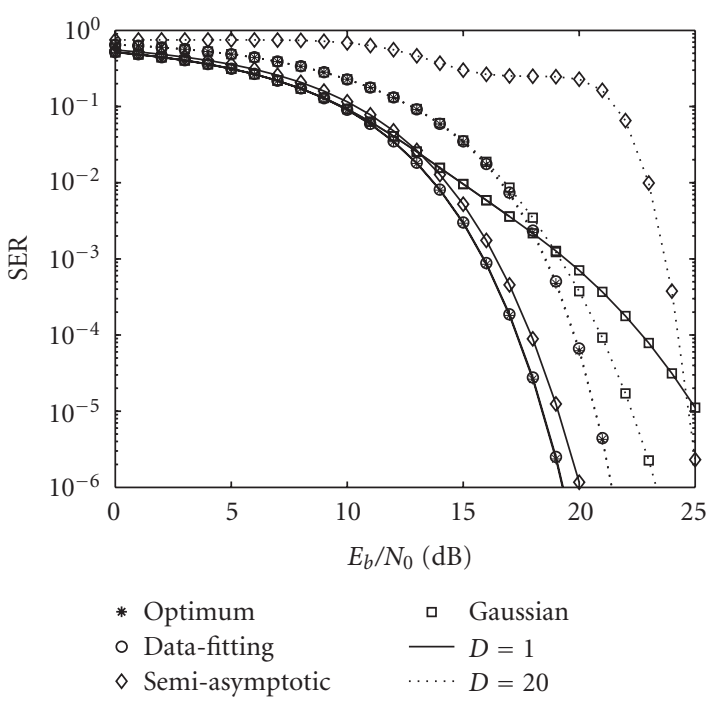

FIGURE 2: SER comparison of different threshold selection schemes $(M=4)$.

fitting threshold selection scheme for an $M$-ary ED-PAM system as

$$
\rho_{m, \text { dat }}=\left\{\begin{array}{l}
E_{1} / 4+N_{0}\left(\sqrt{D-1} K_{0}+D\right), \quad m=0, \\
\left(E_{m}+E_{m+1}\right) / 2+N_{0}\left(K_{m}+D\right), \quad m>0,
\end{array}\right.
$$

where $K_{m}=k_{m, V_{m}} x_{m}^{V_{m}}+k_{m, V_{m}-1} x_{m}^{V_{m}-1}+\cdots+k_{m, 2} x_{m}^{2}+$ $k_{m, 1} x_{m}+k_{m, 0}$ is the polynomial with order $V_{m}$ for the $m$ th threshold, $x_{m}=E_{m+1} / N_{0}$ is the received SNR of the $(m+1)$ th symbol, and $k_{m, v}$ are the coefficients of the polynomial. The coefficients, which minimize (6), are generated by applying the well-known least squares curve-fitting method [10, page 528] for the target SNR range. In Figure 2, we plot the SER as a function of the SNR per bit for the quaternary EDPAM system using (3) now with the actual threshold values determined by four different threshold selection schemes. In this example, $V_{0}=2, V_{1}=1, V_{2}=1$ are adopted in (7). For a comparison we apply the Gaussian approximation scheme presented in [4] to determine each $m$ th threshold $(m=$ $0,1,2)$ separately. We found that the polynomial order has the highest effect on the first threshold and that the selection of $D$ has a significant impact on the relative performance of the schemes. However, the data-fitting scheme performs well for a wide value range of $D$. More detailed reasoning is given in [2].

\section{Conclusion}

We have presented some useful guidelines between a systematic threshold mismatch and the corresponding margin in the average SNR per bit to preserve a desired error probability in a multilevel ED-PAM system. In the case where the increase of the error probability or average SNR is not tolerable, we have proposed a novel near-optimal multilevel threshold selection scheme based on a closed form polynomial data-fitting approach. We have shown that 
the proposed scheme is robust for a wide range of system parameters whereas other suboptimal schemes assume a more restricted value range for the DOFs to work properly. The results can be extended to slowly fading channels and they can be applied, for example, for a high-speed impulse radio.

\section{Acknowledgments}

This work has been mainly carried out in the projects Pervasive Ultra-wideband Low Spectral Energy Radio Systems (PULSERS) and Coexisting Short Range Radio by Advanced Ultra-Wideband Radio Technology (EUWB) which have been partly funded by the European Union. The authors are thankful to Stephane Paquelet and Alexis Bisiaux for the discussions on the energy detection principle. This work is based on the work by Anttonen et al., which appeared in ICUWB '08, 2008IEEE. The paper received the Best Student Paper Award.

\section{References}

[1] H. Urkowitz, "Energy detection of unknown deterministic signals," Proceedings of the IEEE, vol. 55, no. 4, pp. 523-531, 1967.

[2] A. Anttonen, A. Mämmelä, and A. Kotelba, "Sensitivity of energy detected multilevel PAM systems to threshold mismatch," in Proceeedings of the IEEE International Conference on Ultra-Wideband (ICUWB '08), vol. 1, pp. 165-168, Hannover, Germany, September 2008.

[3] G. J. Foschini, L. J. Greenstein, and G. Vannucci, "Noncoherent detection of coherent lightwave signals corrupted by phase noise," IEEE Transactions on Communications, vol. 36, no. 3, pp. 306-314, 1988.

[4] P. A. Humblet and M. Azizoglu, "On the bit error rate of lightwave systems with optical amplifiers," Journal of Lightwave Technology, vol. 9, no. 11, pp. 1576-1582, 1991.

[5] A. Al-Dweik and F. Xiong, "Frequency-hopped multipleaccess communications with noncoherent $M$-ary OFDMASK," IEEE Transactions on Communications, vol. 51, no. 1, pp. 33-36, 2003.

[6] S. Paquelet, L.-M. Aubert, and B. Uguen, "An impulse radio asynchronous transceiver for high data rates," in Proceedings of the International Workshop on Ultra Wideband Systems; Joint with Conference on Ultra Wideband Systems and Technologies (IWUWBS '04), pp. 1-5, Kyoto, Japan, May 2004.

[7] V. I. Kostylev, "Energy detection of a signal with random amplitude," in Proceedings of the IEEE International Conference on Communications (ICC '02), vol. 3, pp. 1606-1610, New York, NY, USA, April-May 2002.

[8] S. M. Kay, Fundamentals of Statistical Signal Processing, Volume 2: Detection Theory, Prentice Hall, Upper Saddle River, NJ, USA, 1998.

[9] P. E. Cantrell and A. K. Ojha, "Comparison of generalized Q-function algorithms," IEEE Transactions on Information Theory, vol. 33, no. 4, pp. 591-596, 1987.

[10] W. Press, B. Flannery, S. Teukolsky, and W. Vetterling, Numerical Recipes in C: The Art of Scientific Computing, Cambridge University Press, Cambridge, Mass, USA, 1988. 

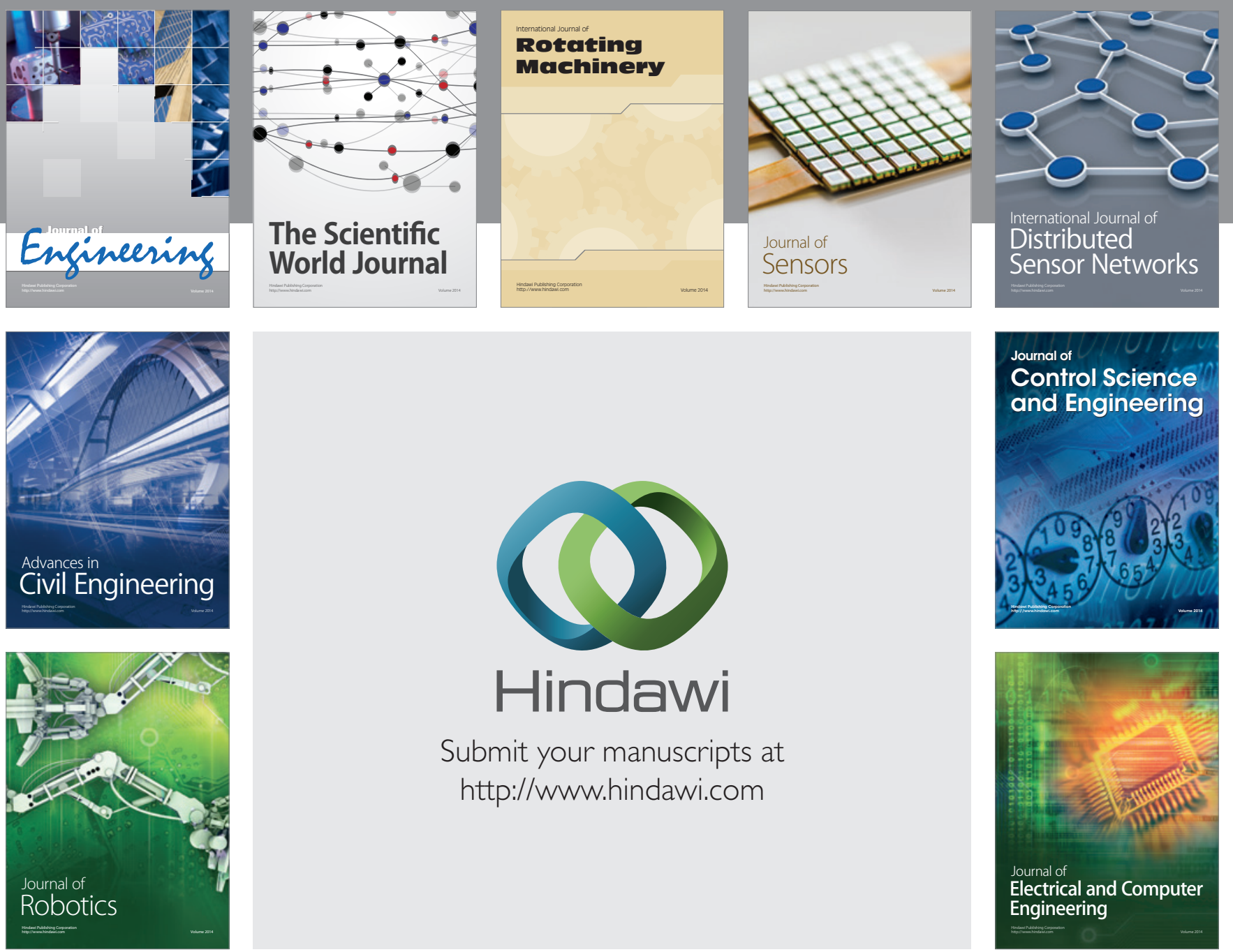

Submit your manuscripts at

http://www.hindawi.com
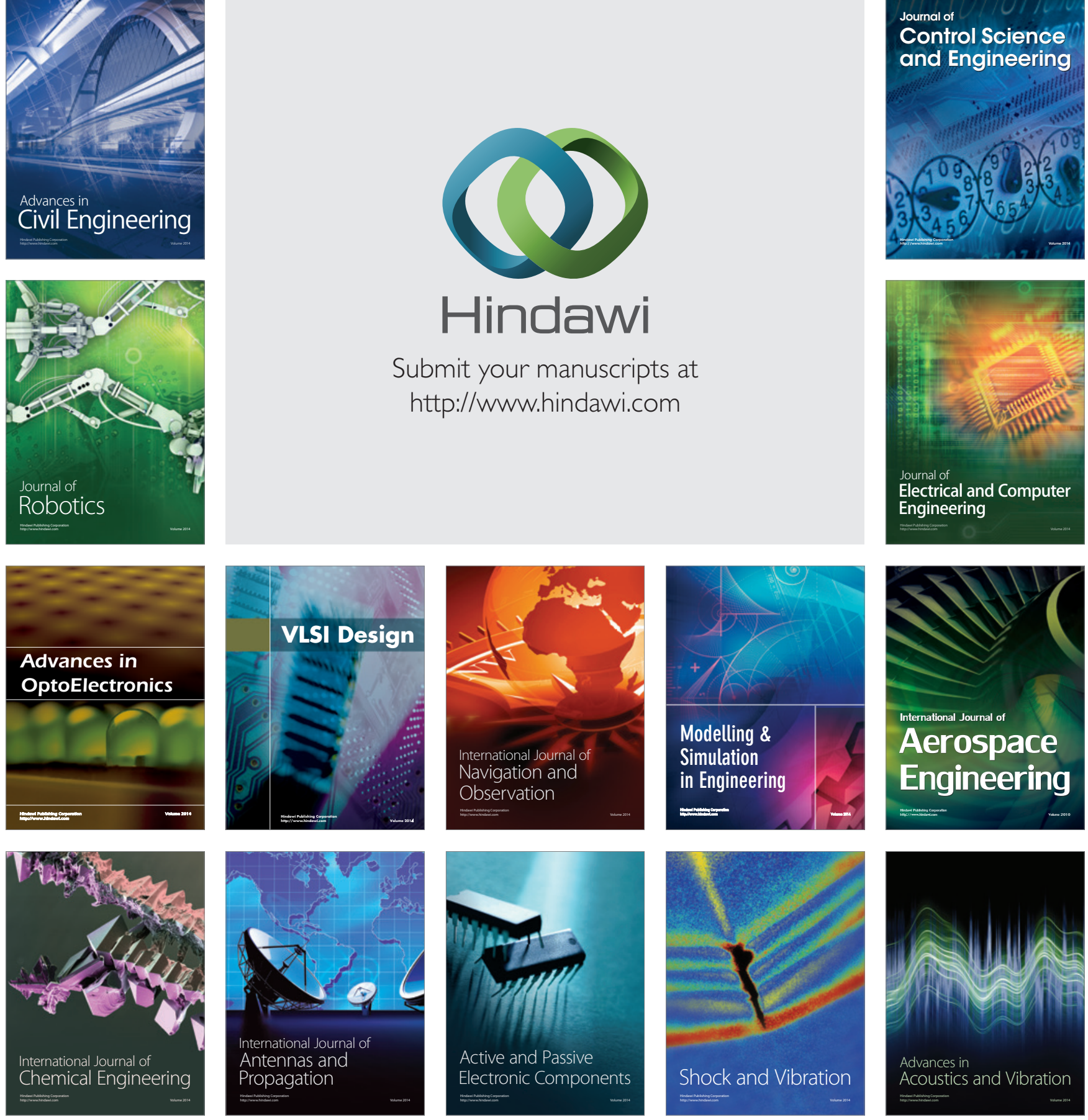